\title{
Ecological study and risk mapping of visceral leishmaniasis in an endemic area of Iran based on a geographical information systems approach
}

\author{
Abdoreza Salahi-Moghaddam¹, Mehdi Mohebali², Ali Moshfae ${ }^{2}$, Majid Habibi², \\ Zabiholah Zarei ${ }^{4}$ \\ ${ }^{1}$ Department of Pathobiology, Bandar Abbas Faculty of Medicine, Hormozgan University of Medical \\ Sciences (HUMS), Iran; ${ }^{2}$ Department of Medical Parasitology and Mycology, Tehran University of Medical \\ Sciences, Tehran, Iran; ${ }^{3}$ Climatological Research Institute, Tehran, Iran; ${ }^{4}$ Meshkin-Shahr Research and \\ Training Center, Tehran University of Medical Sciences, Tehran, Iran
}

\begin{abstract}
Between 1998 and 2001, a total of 1,062 human cases of visceral leishmaniasis were reported from the rural district of Meshkin-Shahr in the mountainous, north-western Iranian province of Ardabil. In the summer of 2008, a cross-sectional study of dogs was conducted in this endemic area by randomly selecting 384 animals from 21 villages and testing them serologically for leishmaniasis. Villages, in which more than $10 \%$ of investigated dogs showed antiLeishmania titres $\geq 1 / 320$, were considered to be high-risk environments. Regression analysis showed no statistically significant correlation between topographic conditions and the prevalence of positive cases. However, when the results were compared with past meteorological records, a statistically significant positive correlation $(P=0.007)$ was found between the number of infected dogs with anti-Leishmania titres $\geq 1 / 640$ and the number of days in a year with temperatures below $0{ }^{\circ} \mathrm{C}$. While humidity showed an inverse correlation $(\mathrm{P}=0.009)$ with the anti-Leishmania titres, a positive correlation $(\mathrm{P}<0.001)$ was found in relation to the amount of rainfall. Mapping of the areas at risk for kala-azar in the Meshkin-Shahr district supports the impression that the low temperatures prevalent in the Ardebil province constitute an important factor influencing the distribution of leishmaniasis there.
\end{abstract}

Keywords: geographical information system, leishmaniasis, mapping, temperature, humidity, ecology, Iran.

\section{Introduction}

Cases of visceral leishmaniasis (VL) are frequent in several parts of Iran. Examples of areas with reported VL include the Meshkin-Shahr district in the Ardabil province (Mohebali et al., 2006) as well as the Azar-Shahr district in the province of East Azarbaijan (Mirsamadi et al., 2003) in the North. Cases are also reported from the Firuz-Abad district in the Fars province in the South (Hashemi-Nasab et

Corresponding author:

Abdoreza Salahi-Moghaddam

Bandar Abbas Faculty of Medicine, Department of Pathobiology

Motahari st, Hormozgan 79149-64153, Iran

Tel. +98 917761 9404; Fax +98 7613330612

Email: asmoghaddam@yahoo.com al., 1980), and in some nomadic communities in other parts of the country (Mohebali et al., 2001). The parasitic agent in the area is Leishmania infantum LON 49 (Mohebali et al., 2002, 2004).

The Meshkin-Shahr district (Fig. 1), a rural, mainly agricultural area, is one of the most important endemic zones for VL in Iran (Soleimanzadeh et al., 1993). Out of 1,050 cases of VL occurring in Iran during the period 1985-1990, 800 (76\%) originated in this district (Soleimanzadeh et al., 1993). Climatologically, Meshkin-Shahr is generally cold, i.e. the average daily temperature is below $0{ }^{\circ} \mathrm{C}$ for more than a third of the year. Dry cattle faeces is traditionally burnt to produce heat at times of low outdoor temperatures. To that end, the faeces from the cattle are processed in blocks and stored in 


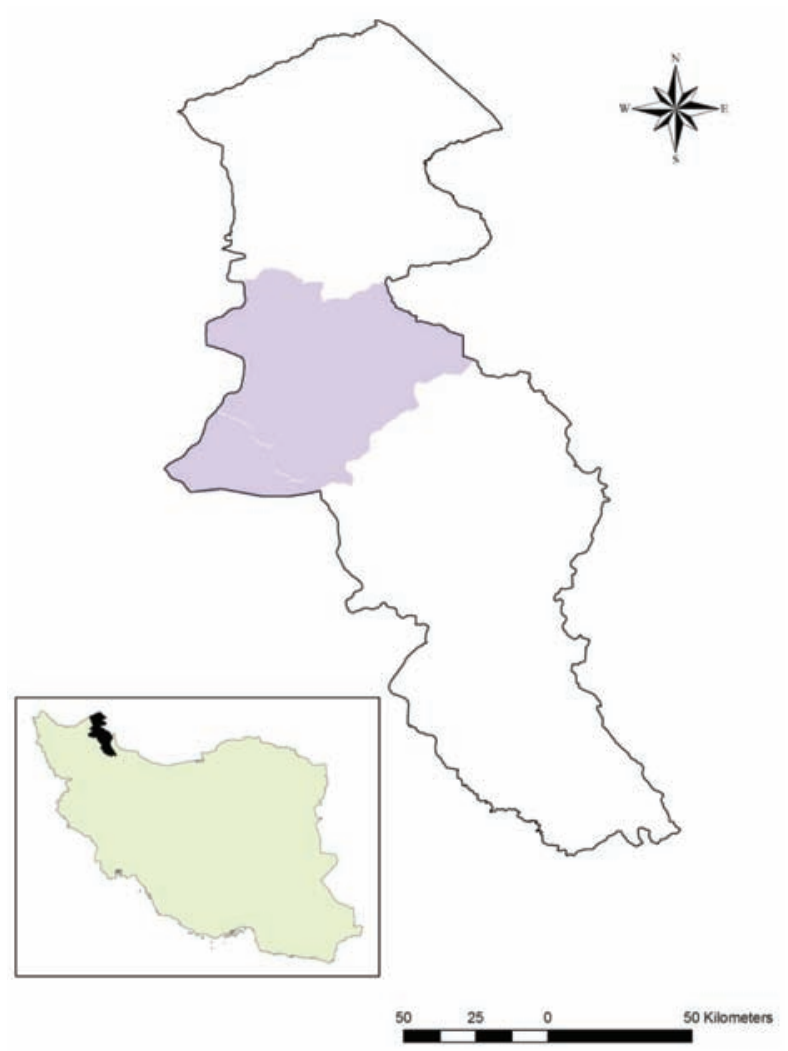

Fig. 1. Maps showing the location of the Ardebil province in Iran and the endemic zone for visceral leishmaniasis (VL) in the Meshkin-Shahr district (violet).

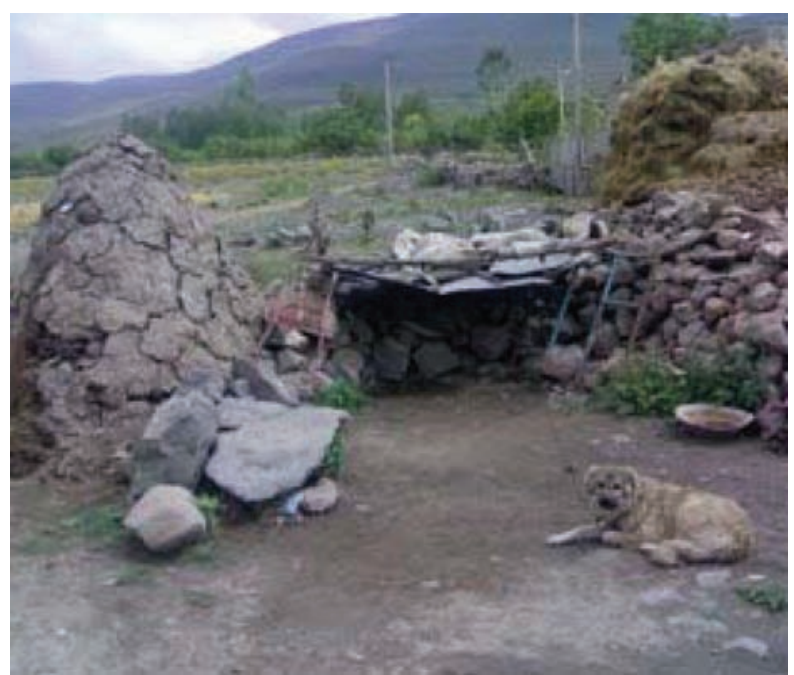

Fig. 2. A dog close to a "galakh" (left) and its summer shelter (right). large conical structures called "galakhs" in local language. However, these structures not only provide an excellent niche for the sand fly vector of the Leishmania parasite, but are also commonly used as dog-shelters in the winter (Fig. 2). In our opinion, it is therefore probable that these "galakhs" play a role in the local epidemiology of VL. In addition, in contrast to other parts of Iran, people in the north-western provinces have a close relation to dogs, which are not only used for shepherding and guarding but also kept as pets. The risk for infection is obvious when one considers that the dog constitutes the predominant host for the Leishmania parasite and that dogs constitute the main reservoir for the disease (Gavgani et al., 2002; Mohebali et al., 2005).

Many Iranian studies have focused on the zoonotic aspects of VL and the role of reservoir hosts for its transmission (Edrissian et al., 1988; Mohebali et al., 2005). Although a geographical information system (GIS) lends itself for the mapping of high-risk, endemic areas for infectious and parasitic diseases (Elnaiem et al., 2003; Rinaldi et al., 2006), no efforts have been made with regard to VL epidemiology in the endemically important Meshkin-Shahr district. In order to better understand the ecology of the disease, and the variables influencing its distribution, we applied GIS with satellite imagery for complementary information as pioneered in India (Sudhakar et al., 2006; Bhunia et al., 2010). The terrestrial data, including parameters such as elevation, temperature and rainfall were collected with the overall aim to facilitate mapping high-risk areas in this district.

\section{Materials and methods}

\section{Study site}

The Ardabil province has a cold, predominantly dry climate. Until recently, this province belonged to Azerbaijan and scientific articles, depending when they were written, may therefore refer to Ardabil as part of either the one or the other country. The investigation presented here was conducted in 2007 and 200 in the Meshkin-Shahr district, which is 
located in the central northern part of the Ardebil province between longitudes $47^{\circ} 19^{\prime}$ and $48^{\circ} 17^{\prime}$ East and latitudes $38^{\circ} 57^{\prime}$ and $38^{\circ} 13^{\prime}$ North (Fig. $1)$. The relative humidity in this area fluctuates between $61 \%$ and $70 \%$ (Fig. 3 ) and the annual rainfall varies between 300 and $385 \mathrm{~mm}$ (Fig. 4). The district includes 416 officially registered localities such as villages and rural areas.

As there is no definitive basis for estimating the VL risk (Ashford, 1999), villages were arbitrarily considered endemic if more than $10 \%$ of sampled dogs test positively for anti-Leishmania antibodies with a serum dilution of $\geq 1 / 320$. Inspections of the general life style and human-dog relationship were carried out on site to gain a better understanding of the ecology of the disease. In the endemic villages,

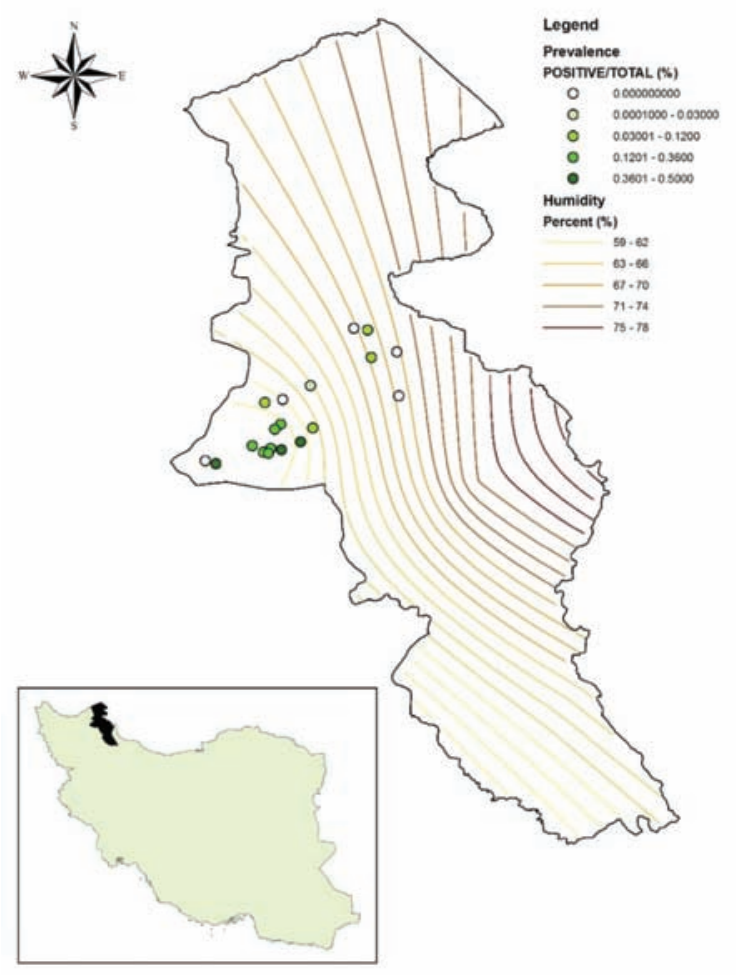

$50 \quad 25 \quad 0 \quad 50$ Kilometers

Fig. 3. Distribution of positive cases for canine leishmaniasis in the Ardebil province overlaid on a map indicating the variation of relative humidity over the territory. the number of roofed houses and the date of infection (when known) were collected, while the environment was recorded from a purely geographic point of view using a global positioning system (GPS) instrument. Villages, considered non-endemic according to previous studies (Soleimanzadeh et al., 1993) were not investigated at this time.

\section{Data collection}

Records of human and animal cases from the period 2003-2008 in the study area were retrieved for eight villages from primary health care files. Meteorological data, including relative humidity, annual rainfall, the state of local rivers, elevation and the number of days in the year, colder than $0{ }^{\circ} \mathrm{C}$

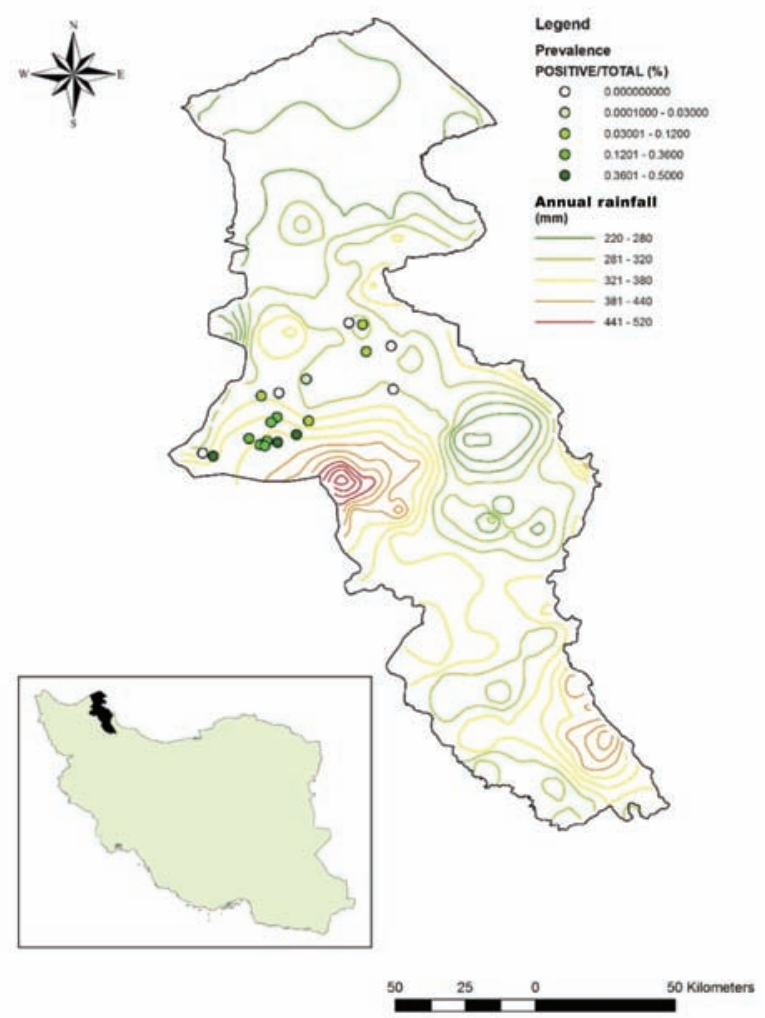

Fig. 4. Distribution of positive cases for canine leishmaniasis in the Ardebil province overlaid on a map indicating the variation of rainfall ( $\mathrm{mm} /$ year) over the territory. 
(freezing days), were retrieved from the Iranian Atmospheric Science and Climatological Research Center (IASCRC).

ArcGIS, version 9.2 (http://www.esri.com/arcgis) was used for the spatial analysis. The information from IASCRC was added to the terrestrial data previously collected and saved in the form of shape-files. Demographic information and data on elevations and other facts such as the location of villages and borders were used as shape-file base-layers. We used ArcGIS for classifying areas as either safe (0) or as posing a degree of risk for infection (1) based on the cut-off values found (see Figs. 3-5), e.g. humidity less than $63 \%$ was counted as safe $(0)$, while higher recordings were given a (1) designation. Likewise, areas with an annual rainfall exceeding $340 \mathrm{~mm}$ were recorded as (1), while areas with less rain received a (0) value. Based on this information, raster maps were produced for each of the four critical parameters studied, i.e. rainfall, humidity, freezing days and elevation (Figs. 3-5). These maps were finally combined in a way that summed up the four critical threshold values in such a way that areas could be differentiated using a scale ranging from 0 to 4, the lowest value signifying no risk at all with the others suggesting various degrees of risk, i.e. if three abovethreshold values had been found (e.g. high rainfall, low humidity and a high number of freezing days), the zone was designated as (3) and so on.

The SPSS statistics package version 13, including $\chi^{2}$ and ANOVA, was used for regression analysis and other statistical analyses, while co-kriging was applied with the variables as datasets to produce risk maps. Those variables which had correlation with endemic areas and their cut-off values were calculated using SPSS software.

To appraise the general distribution of canine leishmaniasis in the district under study, we undertook serological testing of 384 local dogs, randomly selected from 21 villages in the endemic area, using the direct agglutination test (DAT) and a Leishmania antigen produced from promastigotes isolated from the spleens of infected, domestic dogs and grown in RPMI 1640 medium (Moshfe et al., 2008).

\section{Results}

The prevalence of canine leishmaniasis showed an indirect correlation with humidity $(\mathrm{P}=0.009)$. The threshold was found to be a relative humidity of $63 \%(\mathrm{P}=0.008)$ depicted by the cases of leishmaniasis overlaid on a map showing the variation in humidity in the Ardabil province (Fig. 3). In addition, a direct correlation was seen between rainfall and positive cases $(\mathrm{P}<0.001)$ with the strongest correlations seen in areas receiving more than $340 \mathrm{~mm}$ rain per year (Fig. 4). As seen in the figure, the difference with reference to the number of cases in areas characterised by relatively little rain as compared to areas with much rain is obvious $(\mathrm{P}<0.001)$.

There seemed to be no significant correlation between elevation and prevalence of positive cases in the regression analysis. However, after grouping data, considering the $1,125 \mathrm{~m}$ level of elevation as critical and the 1/640 titer as the cut-off level, there was indeed a significant correlation $(\mathrm{P}=0.038)$.

Assessment of VL infection in the houses, using a $\chi^{2}$ test, showed a statistically highly significant correlation $(\mathrm{P}<0.001)$ between human cases and infected dogs in the past 5 years, i.e. in each house where a canine case was reported, a human case appeared within this time-span. Regression analysis of available data showed that although $10-12{ }^{\circ} \mathrm{C}$ is a suitable average temperature for the transmission of $\mathrm{VL}$, there was no significant correlation between temperature and positive cases. In the Ardebil province, the number of days below $0{ }^{\circ} \mathrm{C}$ varied from 94 to 119 days in the year. We found that the number of freezing days in the year had a direct correlation with positive serology in the $\operatorname{dogs}(\mathrm{P}=$ 0.007). The villages with more than 107 days per year below $0{ }^{\circ} \mathrm{C}$ had a significantly higher prevalence of dogs testing positively $(\mathrm{P}=0.003)$. We considered 107 as the threshold for this variable. Fig. 5 shows the distribution of villages in the different zones.

Putting the collected information together made it possible to map the high-risk, endemic areas in the Ardebil province. Fig. 6 shows the results for vil- 
lages using the co-kriging method, while Fig. 7 shows the risks resulting from summing-up the different risk parameters. Based on the national demographic information, it could be calculated that about 29,000 people live in high-risk areas for Leishmania infection (grade 4), about 128,000 people live in medium-risk areas (grade 3 ) and 142,500 people in low-risk (grade 2) areas.

\section{Discussion}

This study is based on the assumption that infected local dogs, which live in close relationship with households, would be highly likely to transmit Leishmania to humans via the available sandfly vector. Although we are well aware that an in-depth study would need to also explore the extent of

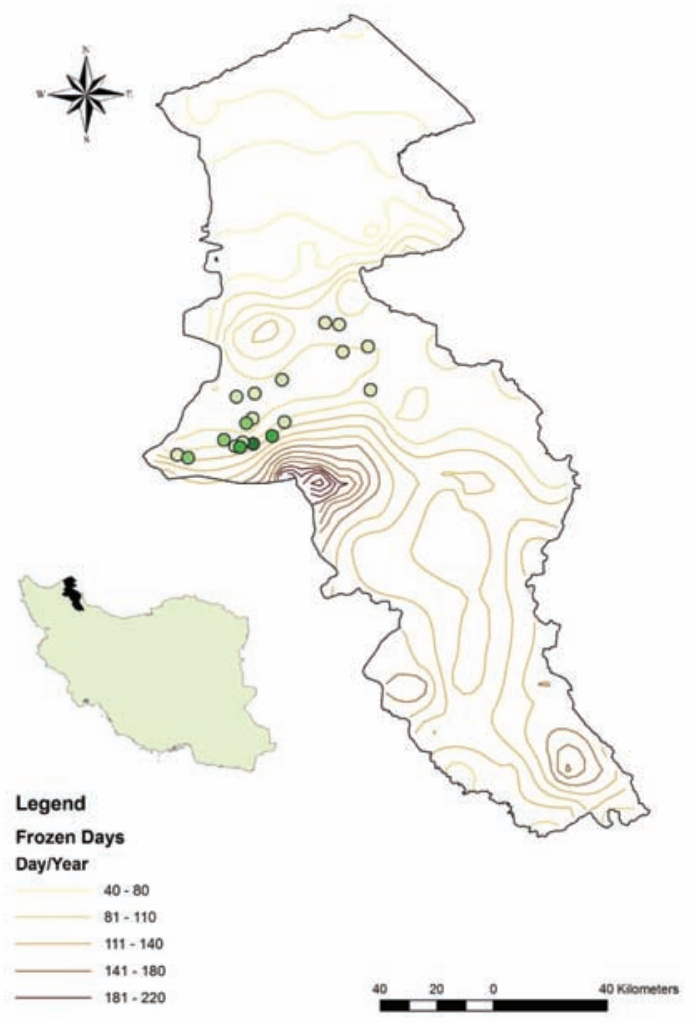

Fig. 5. Distribution of positive cases for canine leishmaniasis in the Ardebil province overlaid on a map indicating the variation over the territory of how many days per year stay below $0{ }^{\circ} \mathrm{C}$ on avergage.

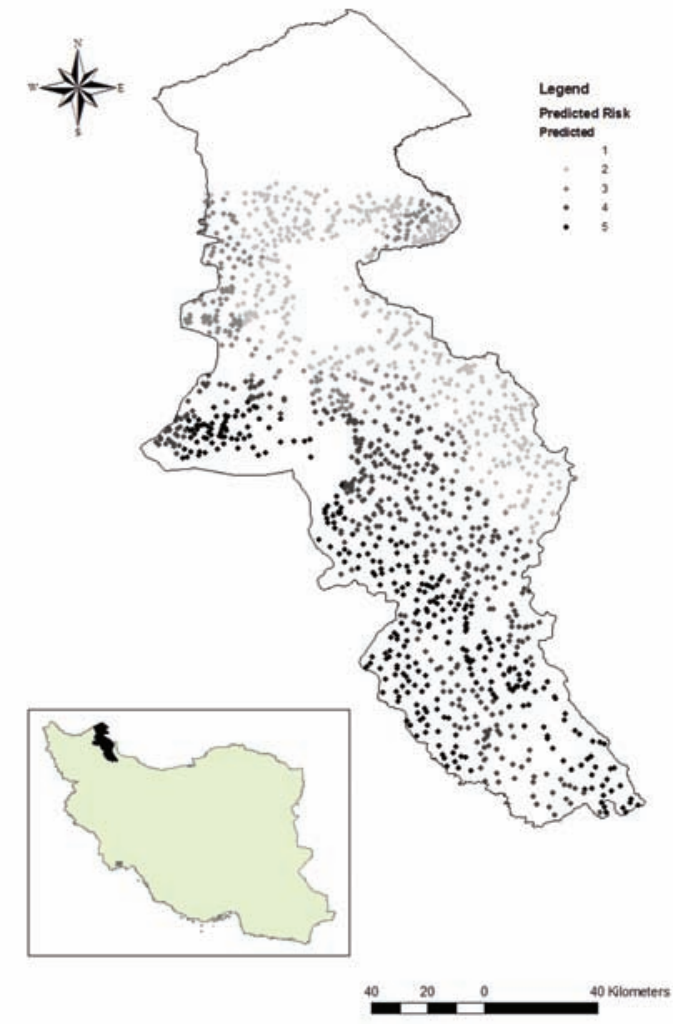

Fig. 6. Risk-map for canine leishmaniasis in the Ardebil province constructed using the co-kriging method.

human infection, it was felt that the serological testing of the dogs can be used as a proxy for endemicity as the first step in a wider investigation.

Studies aiming at an improved understanding of the ecology of VL have been conducted in other parts of the world. For example, Bavia et al. (2005) has shown the importance of the low level of the normalized difference vegetation index (NDVI) in Brazil for the prevalence of both human and canine VL. These findings are corroborated by the observations reported in the northern part of India by Bhunia et al. (2010). Our findings with respect to rainfall and endemicity correspond with reports from eastern Sudan (Elnaiem et al., 2003). However, while the Sudanese studies found altitude together with rainfall particularly useful for risk mapping, our study found that the number of days below 


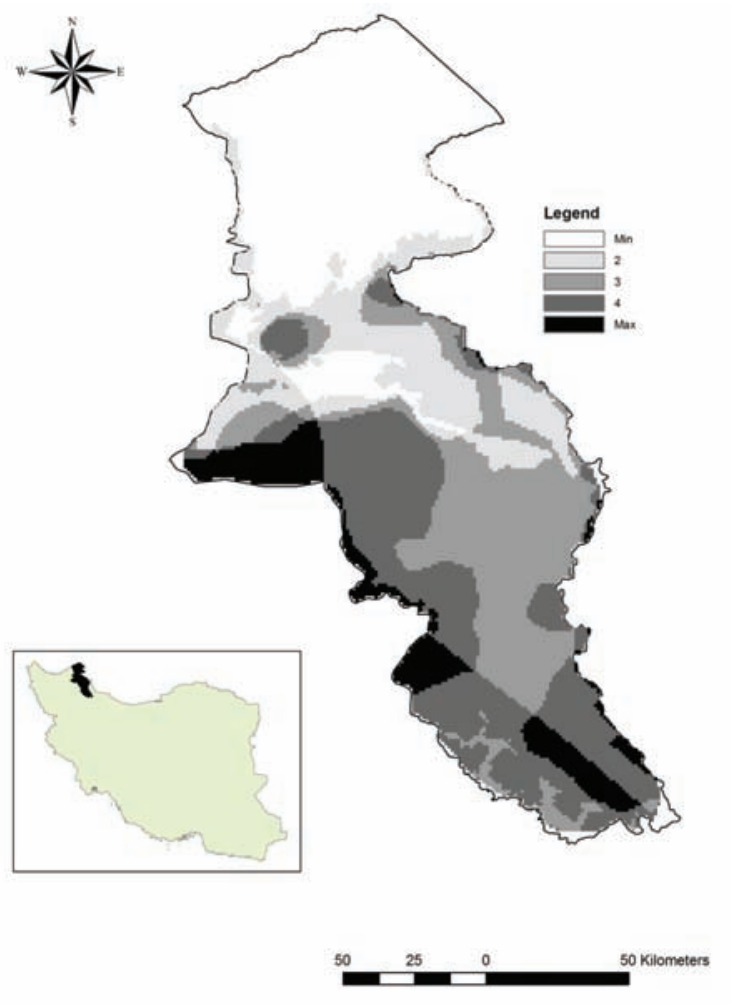

Fig. 7. The different zones according to the relative risk for canine leishmaniasis according to four climatic parameters.

freezing, rainfall and humidity taken together worked even better as predictors. Indeed, while we also note the influence of humidity, our criteria are quite similar to those reported by Elnaiem et al. (2003) if cold days and high altitude are taken as expression of the same variable. Even if this variable is lower in the Ardabil province than in many other areas, it should be noted that a very high percentage of humidity $(\geq 80 \%)$ is often recorded in Gilan, the neighbourhood province.

While Bhunia et al. (2010) found a negative correlation with higher altitudes, only relatively low altitudes, where temperatures never reached freezing, were investigated. However, also in our study, higher altitudes would have been expected to result in lower risk of infection. Indeed, the higher the positive correlation between prevalence of VL and the numbers of days below freezing is in conflict both with the biology of the insect vector and the ecology.
On the other hand, we feel that there are reasons to believe that the "galakhs" influence the overall situation by providing a warm shelter during cold days and nights in this area. While these "galakhs" represent a cold day's shelter they also provide a local ecological niche for sandfly/dog interaction. It must, however, be pointed out that no entomological study have been carried out to prove this hypothesis. Hence, investigations of the importance of these shelters for leishmaniasis are needed to better understand the correlation found between freezing and risk. The correlation of VL with rainfall, humidity and elevation and its higher prevalence at low temperatures are important findings for mapping highrisk areas in the north-western part of Iran.

\section{Conclusions}

Satellite imagery, complemented with a GIS database, is useful for the study of leishmaniasis since geographical parameters play an important role in evaluating the risk for its distribution. Although the spread of this disease is highly correlated with certain ranges of temperature, rainfall, humidity and NDVI, paradoxical endemic situations may appear when prevailing economy and social habits conspire to create special conditions favouring transmission.

\section{Acknowledgments}

This manuscript was prepared with the kind cooperation of Mr. Rasul Hemati, Dean of Ardebil Province Meteorology Center. We do appreciate all his cooperation. This research was funded by Hormozgan University of Medical Sciences (HUMS), Tropical Disease Research Center.

\section{References}

Ashford RW, 1999. Leishmaniasis. Protozoal disease. HM Gilles ed. Oxford University Press 1. New York, USA, pp. 407.

Bavia ME, Carneiro DD, Gurgel HC, Madureira-Filho C, Barbosa MG, 2005. Remote sensing and geographical information systems and risk of American visceral leishma- 
niasis in Bahia, Brazil. Parassitologia 47, 165-169.

Bhunia GS, Kesari S, Jeyaram A, Kumar V, Das P, 2010. Influence of topography on the endemicity of Kala-azar: a study based on remote sensing and geographical information system. Geospat Health 4, 155-165.

Edrissian GH, Hafizi A, Afshar A, Soleiman-Zadeh G, Movahed-Danesh AM, Garoussi A, 1988. An endemic focus of visceral leishmaniasis in Meshkin-Shahr, east Azerbaijan province, north-west part of Iran and IFA serological survey of the disease in this area. Bull Soc Path Exot 81, 238-248.

Elnaiem DEA, Schorscher J, Bendall A, Obsomer V, Osman ME, Mekkawi AM, Connor SJ, Ashford RW, Thomson MC, 2003. Risk mapping of visceral leishmaniasis: the role of local variation in rainfall and altitude on the presence and incidence of kala-azar in eastern Sudan. Am J Trop Med Hyg 68, 10-17.

Gavgani AS, Mohite H, Edrissian GH, Mohebali M, Davies CR, 2002. Domestic dog ownership in Iran is a risk factor for human infection with Leishmania infantum. Am J Trop Med Hyg 67, 511-515.

Hashemi-Nasab A, Zadeh-Shirazi H, 1980. Visceral leishmaniasis (kala-azar) in Fars province, Iran: study of 130 cases. J Trop Med Hyg 83, 119-122.

Mirsamadi N, Mohebali M, Attari MR, Edrissian GH, 2003. Serological survey on visceral leishmaniasis (Kala-azar) in Azar-Shar, Azarbaijan province, northwest of Iran. Hakim Res J 6, 17-22.

Mohebali M, Edrissian GH, Nadim A, Hajjaran H, Akhoundi B, Hooshmand B, Zarei Z, Arshi S, Mirsamadi N, Manouchehri Naeini K, Mamishi S, Sanati AA, Moshfeh AA, Charehdar S, Fakhar M, 2006. Application of direct agglutination test (DAT) for the diagnosis and seroepidemiological studies of visceral leishmaniasis in Iran. Iranian J Parasitol 1, 15-25.
Mohebali M, Hajjaran H, Hamzavi Y, Mobedi I, Arshi S, Zarei Z, Akhoundi B, Naeini KM, Avizeh R, Fakhar M, 2005. Epidemiological aspects of canine visceral leishmaniosis in the Islamic Republic of Iran. Vet Parasitol 129, 243-251.

Mohebali M, Hamzaviy Y, Forouzani AR, Edrissian GH, 2001. Seroepidemiological studies of visceral leishmaniasis in Bushehr province, south of I.R. Iran. Eastern Mediterr Health J 7, 912-917.

Mohebali M, Javadian E, Yaghoobi-Ershadi MR, Akhavan AA, Hajjaran H, Abaei MR, 2004. Characterization of Leishmania infection in rodents from endemic areas of the Islamic Republic of Iran. Eastern Mediterr Health J 10, 591-599.

Mohebali M, Motazedian MH, Parsa F, Hajjaran H, 2002. Identification of Leishmania species from different parts of Iran using a random amplified polymorphic DNA in human, animal reservoirs and vectors. Med J Islamic Republic Iran 15, 243-246.

Moshfe AA, Mohebali M, Edrissian GH, Zarei Z, Akhoundi B, Kazemi B, Jamshidi SH, Mahmoodi M, 2008. Seroepidemiological study on canine visceral leishmaniasis in Meshkin-Shahr district, Ardebil province, northwest of Iran during 2006-2007. Iranian J Parasitol 3, 1-10.

Rinaldi L, Musella V, Biggeri A, Cringoli C, 2006. New insights into the application of geographical information sistems and remote sensing in veterinary parasitology. Geospat Health 1, 33-47.

Soleimanzadeh GE, Movahhed-Danesh AM, Nadim A, 1993. Epidemiological aspects of kala-azar in MeshkinShahr, Iran: human infection. Bull World Health Organ 71, 759-762.

Sudhakar S, Srinivas T, Palit A, Kar SK, Battacharya, SK, 2006. Mapping of risk prone areas of kala-azar (visceral leishmaniasis) in parts of Bihar State, India: an RS and GIS approach. J Vector Borne Dis 43, 115-122. 